I.Korchagina

Nagoya Math. J.

Vol. 171 (2003), 197-206

\title{
ON A THEOREM OF P. FONG
}

\author{
INNA KORCHAGINA
}

\begin{abstract}
This paper is a contribution to the "revision" project of Gorenstein, Lyons and Solomon, whose goal is to produce a unified proof of the Classification of Finite Simple Groups.
\end{abstract}

$\S 1$.

This paper is a contribution to the "revision" project of Gorenstein, Lyons and Solomon, whose goal is to produce a unified proof of the Classification Theorem of Finite Simple Groups [GLS]. Theorem $\mathcal{C}_{2}$ [GLS2] is the part of the proof of the Classification Theorem which deals with the "small odd cases". One case of this theorem is the following result:

THEOREM. If $G$ is a finite simple group of odd type and of 2-rank 3 (where the 2-rank of $G$ is a 2-rank of a Sylow 2-subgroup of $G$ ), then one of the following holds:

(1) $G \cong{ }^{2} G_{2}(q)$ for some $q=3^{2 n+1}, n \geq 1$;

(2) $G \cong G_{2}(q)$ for some odd $q$ with $q>3$;

(3) $G \cong{ }^{3} D_{4}(q)$ for some odd $q$; or

(4) $G \cong M_{12}, J_{1}$ or $O N$.

In order to prove this theorem, one begins by showing that $G \approx G^{*}$ for some $G^{*} \in\left\{{ }^{2} G_{2}(q), G_{2}(q),{ }^{3} D_{4}(q), M_{12}, J_{1}, O N\right\}$ with $q$ odd, which means that the following conditions hold:

(1) $G$ and $G^{*}$ have isomorphic Sylow 2-subgroups;

(2) $G$ has exactly one class of involutions $z^{G}$; and

(3) If $C=C_{G}(z)$, then $C \cong C_{G^{*}}\left(z^{*}\right)$ for $z^{*}$ an involution of $G^{*}$.

Received February 25, 2002.

2000 Mathematics Subject Classification: 20E32. 
At this time the proof splits into two major cases. The first one deals with the situation $G^{*}={ }^{2} G_{2}(q)$. In the second case, $C_{G}(z)$ has a subgroup $K$ of index 2 with $K=K_{1} \circ K_{2}$ and $K_{i} \cong S L_{2}\left(r_{i}\right)$ (i.e., $\left[K_{1}, K_{2}\right]=1$, $\left.K_{1} \cap K_{2}=Z(K)=Z\left(K_{i}\right)=\langle z\rangle\right)$, where $r_{2}=q$ and $r_{1}=q$ or $q^{3}$. The analysis depends on the values of the parameters $r_{1}$ and $r_{2}$. If $r_{1}>r_{2}$ or $r_{1}=r_{2} \neq 3^{n}$, then local analysis shows that $G \cong 3 D_{4}(q)$ or $G_{2}(q)$. Finally, suppose that $q=r_{1}=r_{2}=3^{n}$ with $n \geq 2$. The crucial point of the analysis is to show that the centralizer of the central involution does not contain a Sylow 3-subgroup of $G$. If $q>9$, this is a fairly easy application of an order formula obtained by Brauer using modular character theory. This was proved by Fong and Wong [FW]. Unfortunately for the case $q=9$, this proof does not work. One has to try to come up with a different trick. This is achieved in the theorem which we state:

THEOREM 1.1. There is no finite group G satisfying the following conditions:

(1) G has a unique conjugacy class of involutions;

(2) If $z$ is an involution of $G$, then $C_{G}(z)=\left(L_{1} \circ L_{2}\right) T$, where $L_{i} \cong$ $S L_{2}(9), T \cong \mathbb{Z}_{2}$ (i.e., $\left[L_{1}, L_{2}\right]=1$ and $\left.L_{1} \cap L_{2}=\langle z\rangle\right)$ and $C_{G}(z) / L_{i} \cong$ $P G L_{2}(9)$ for $i=1,2$;

(3) For every nontrivial 3-subgroup $P \leq C_{G}(z)$, we have $N_{G}(P) \leq C_{G}(z)$;

(4) Every nontrivial 5-element of $G$ is conjugate to some nontrivial 5element of $L_{1} \cup L_{2}$ and $C_{G}(s) \leq C_{G}(z)$ for all nontrivial 5-elements $s \in L_{1} \cup L_{2} ;$ and

(5) 7 divides the order of $G$.

We remark that (3), (4) and (5) follow by local group theory method from (1), (2) and the hypothesis that $C_{G}(z)$ contains a Sylow 3 -subgroup of $G$ [GLS2]. Thus Theorem 1.1 leads one to the desired goal: $G \cong G_{2}(9)$. This result was first announced by $\mathrm{P}$. Fong in [F1]. If $G \approx G_{2}(9)$, then his proof, an elaborate exercise in exceptional character theory, occupies 25 pages of unpublished notes [F2]. In this paper we give a considerably shorter proof of this result. We begin in the same way as Fong by establishing a group order formula (equation (5) below) using the work of M. Suzuki, but then we apply a theorem of Frobenius in the manner of Lyons [L]. Combining those 
two results with the Chinese Remainder Theorem and Sylow's Theorem, we obtain an easy contradiction, proving the result. We refer the reader to [Co] for the basic terminology and results of exceptional character theory.

We now begin the proof. We assume the contrary and proceed to a contradiction in a sequence of lemmas. Fix a nontrivial involution $z \in G$ and let $C=C_{G}(z)$.

Consider the set $S \subseteq C$ which consists of the following elements:

$(\mathcal{S} 1)$ roots of $z$;

$(\mathcal{S} 2)$ 3-singular elements; and

(S3) non-trivial 5-elements of $L_{1} \cup L_{2}$.

For $s \in S$, we let $C_{G}^{*}(s)=\left\{g \in G \mid s^{g}=s\right\} \cup\left\{g \in G \mid s^{g}=s^{-1}\right\}$.

LEMma 1.2. If $s \in S$, then $C_{G}^{*}(s) \leq C$.

Proof. There are three types of elements in $S$. Let us deal with them one by one. If $s$ is a root of $z$, then clearly $C_{G}^{*}(s) \leq C$. If $s$ is a 3element, then the result follows from the hypothesis of the theorem. But this immediately implies the result for all 3-singular elements. Finally if $s \in S$ is a 5-element, we have the following:

$$
C_{G}^{*}(s) \geq C_{C}^{*}(s) \geq C_{C}(s)=C_{G}(s) .
$$

But $\left|C_{G}^{*}(s): C_{G}(s)\right| \leq 2$, while $\left|C_{C}^{*}(s): C_{C}(s)\right|=2$. Thus $C_{G}^{*}(s) \leq C$.

LEMMA 1.3. $S$ is a closed set of special classes.

Proof. There are four things that we must check:

(1) $S$ is a normal subset of $C$;

(2) Whenever $s \in S$, every generator of $\langle s\rangle$ also lies in $S$;

(3) Whenever $s_{1}$ and $s_{2}$ are elements of $S$ which are conjugate in $G$, then $s_{1}$ and $s_{2}$ are conjugate in $C$; and

(4) If $s \in S$, then $C_{G}(s) \leq C$.

Clearly conditions (1) and (2) follow immediately from the definition of $S$. Condition (4) follows from Lemma 1.2. Finally let us deal with the condition (3). If $s_{1}, s_{2}$ are the roots of $z$ and $h \in G$ is such that $s_{1}=s_{2}^{h}$, then $z^{h}=z$, and so $h \in C$.

Finally suppose that either $s_{1}, s_{2}$ are nontrivial $G$-conjugate 3 -singular elements of $S$, or $s_{1}$ and $s_{2}$ are nontrivial $G$-conjugate 5-elements of $S$. 
Then there exists $h \in G$ with $s_{1}=s_{2}^{h}$ and so $C_{G}\left(s_{1}\right)=C_{G}\left(s_{2}^{h}\right)=C_{G}\left(s_{2}\right)^{h}$. In both cases $\langle z\rangle$ is the unique Sylow 2-subgroup of $Z\left(C_{G}\left(s_{i}\right)\right)$. Hence $\langle z\rangle$ is a characteristic subgroup of $C_{G}\left(s_{i}\right)$ for $i=1,2$. Therefore $\langle z\rangle^{h}=\langle z\rangle$, and so $h \in C$.

COROllary 1.4. Induction is an isometry from the set $\mathcal{M}_{C}(S)$ of class functions of $C$ which vanish outside of $S$ to the character ring $\mathcal{C h}(G)$ of $G$.

Proof. Since $S$ is a closed set of special classes of $C$, the result follows immediately from Theorem 9 in [Co].

LEMMA 1.5. There exists a class function $\theta$ of $C$ such that $\theta \in \mathcal{M}_{C}(S)$ and the following conditions hold:

(1) $(\theta, \theta)_{C}=3$;

(2) $(\theta, \theta)_{C}=\left(\theta^{G}, \theta^{G}\right)_{G} ;$ and

(3) $\left(\theta^{G}, 1_{G}\right)=1$.

Proof. Let us simply construct such a class function. Consider $X_{1} \times X_{2}$ with $X_{i} \cong P G L_{2}(9), i=1,2$. Let $\chi_{i}$ be a Steinberg character of $X_{i}$, $i=1,2$. Then $\chi_{1} \times \chi_{2}$ is an irreducible character of a group isomorphic to $P G L_{2}(9) \times P G L_{2}(9)(4.21[\mathrm{Is}])$. Take the lift of $\chi_{1} \times \chi_{2}$ to the double cover $C^{*}$ of $P G L_{2}(9) \times P G L_{2}(9)$, which contains $C$ as a subgroup of index 2 . Now define $\alpha$ to be the restriction of this lift to $C$, i.e., $\alpha$ is an irreducible character of $C$ of degree 81 with $\operatorname{ker}(\alpha)=\langle z\rangle$.

Let $\rho$ be an irreducible character of $L_{1} \circ L_{2}$ of degree 8 with $\operatorname{ker}(\rho)=L_{2}$ and $\lambda$ be one of the two irreducible characters of $L_{1} \circ L_{2}$ of degree 5 with $\operatorname{ker}(\lambda)=L_{1}$. Denote $\beta=(\rho \cdot \lambda)^{C}$. Then $\beta$ is an irreducible character of $C$ of degree 80 such that $\operatorname{ker}(\beta)=\langle z\rangle$ and $\left.\beta\right|_{L_{1} \circ L_{2}}=\rho \cdot\left(\lambda+\lambda^{\prime}\right)$ where $\lambda^{\prime}$ is the other character of $L_{1} \circ L_{2}$ of degree 5 with $L_{1}$ in its kernel.

Finally consider the following class function: $\theta=1_{C}+\beta-\alpha$. By direct calculations, we see that $\theta$ vanishes outside of $S$. Let us study some properties of $\theta$. Clearly $(\theta, \theta)_{C}=\left(1_{C}+\beta-\alpha, 1_{C}+\beta-\alpha\right)_{C}=3$. Also by Corollary 1.4, $(\theta, \theta)_{C}=\left(\theta^{G}, \theta^{G}\right)_{G}$. Finally, by Frobenius Reciprocity (p.62, $[\mathrm{Is}]),\left(\theta^{G}, 1_{G}\right)=\left(\theta, 1_{H}\right)=1$.

This lemma has very important consequences:

COROllary 1.6. There exist irreducible complex characters $\Psi$, $\Phi$ of $G$ such that $\theta^{G}=1_{G}+\Psi-\Phi$, and the following conditions hold:

(1) $\Phi(1)=1+\Psi(1)$ and $\Phi(z)=1+\Psi(z)$; and

(2) $|\Psi(z)| \leq 509$. 
Proof. Since $\theta^{G}(1)=0$, Lemma 1.5 implies the existence of irreducible complex characters $\Psi, \Phi$ of $G$ such that $\theta^{G}=1_{G}+\Psi-\Phi$. Moreover since $\theta^{G}(z)=0$, condition (1) of the corollary obviously holds.

Finally, $1+\Psi(z)^{2}+\Phi(z)^{2} \leq \sum_{\chi} \chi(z)^{2}$, where the summation is taken over all the irreducible characters of $G$. But $\sum_{\chi} \chi(z)^{2}=|C|$ by Orthogonality Relations (p.21, [Is]). Applying condition (1), we obtain that $1+\Psi(z)^{2}+(\Psi(z)+1)^{2} \leq|C|$ which implies that $|\Psi(z)| \leq 509$.

Next define a complex-valued class function $\xi$ of $G$ by

$$
\xi(h)=\sum_{\chi} \frac{\chi(z)^{2}}{\chi(1)} \chi(h)
$$

where the summation is taken over all the irreducible characters of $G$. Let us use a simple manipulation to present $\xi$ in a slightly different way:

$$
\xi(h)=\frac{|G|}{|C|^{2}} \sum_{\chi} \frac{\chi(z)^{2}}{\chi(1)} \chi(h) \frac{|C|^{2}}{|G|}=a_{z z h} \frac{|C|^{2}}{|G|}=a_{z z}(h) \frac{|C|^{2}}{|G|}
$$

where $a_{z z}: G \rightarrow \mathbf{C}$ is the class function defined for all $h \in G$ by

$$
a_{z z}(h)=a_{z z h}=\left|\left\{\left(h_{1}, h_{2}\right) \in z^{G} \times z^{G}: h_{1} h_{2}=h\right\}\right|
$$

Since $\xi$ is a complex-valued class function on $G$, we may calculate $\left(\theta^{G}, \xi\right)_{G}$ :

$$
\left(\theta^{G}, \xi\right)_{G}=\left(1_{G}+\Psi-\Phi, \sum_{\chi} \frac{\chi(z)^{2}}{\chi(1)} \chi\right)_{G}=1+\frac{\Psi(z)^{2}}{\Psi(1)}-\frac{\Phi(z)^{2}}{\Phi(1)}
$$

Using Corollary 1.6(1), we obtain the following formula:

$$
\left(\theta^{G}, \xi\right)_{G}=1+\frac{\Psi(z)^{2}}{\Psi(1)}-\frac{(\Psi(z)+1)^{2}}{\Psi(1)+1}=\frac{(\Psi(1)-\Psi(z))^{2}}{\Psi(1) \cdot(\Psi(1)+1)}
$$

On the other hand using Frobenius Reciprocity and formula (1.2), we have:

$$
\left(\theta^{G}, \xi\right)_{G}=\left(\theta,\left.\xi\right|_{C}\right)_{C}=\left(\theta,\left.\frac{|C|^{2}}{|G|} a_{z z}\right|_{C}\right)_{C}=\frac{|C|^{2}}{|G|}\left(\theta,\left.a_{z z}\right|_{C}\right)_{C}
$$

Since $\theta$ vanishes outside of $S$, we basically are dealing with $\left.a_{z z}\right|_{S}$. Since $h_{i} s h_{i}=s^{-1}$ for $i=1,2$, we have that $h_{i} \in C_{G}^{*}(s)$. But by Lemma 1.2, if 
$s \in S$, then $C_{G}^{*}(s) \leq C$ and so for every $s \in S$ we have that $a_{z z s}$ can be written as

$$
a_{z z s}=a_{z z s}^{\prime}+a_{z t s}^{\prime}+a_{z l s}^{\prime}+a_{t t s}^{\prime}+a_{t z s}^{\prime}+a_{t l s}^{\prime}+a_{l l s}^{\prime}+a_{l z s}^{\prime}+a_{l t s}^{\prime}
$$

where $a_{z z s}^{\prime}, \ldots, a_{l t s}^{\prime}$ are algebra constants of $C$ with $z, t, l$ being the representatives of all the conjugacy classes of involutions in $C$, for $\left.z^{G}\right|_{C}=$ $\{z\} \cup t^{C} \cup l^{C}$. Notice that the only element of $S$ inverted by $z$ is $z$ itself. Clearly $a_{z h z}^{\prime}=0$ for all $h \in C-\{1\}$. So we must have $a_{z z s}^{\prime}=a_{z t s}^{\prime}=a_{z l s}^{\prime}=$ $a_{t z s}^{\prime}=a_{l z s}^{\prime}=0$. Therefore

$$
a_{z z s}=a_{t t s}^{\prime}+a_{t l s}^{\prime}+a_{l l s}^{\prime}+a_{l t s}^{\prime}
$$

All this allows us to reduce the situation to the calculations inside $C$. So we obtain the following result:

$$
\left(\theta^{G}, \xi\right)_{G}=\frac{2^{14} \cdot 3^{8} \cdot 5^{3} \cdot 41^{2}}{|G|}
$$

Finally combining (1.3) and (1.4) we obtain:

$$
|G|=2^{14} \cdot 3^{8} \cdot 5^{3} \cdot 41^{2} \cdot \frac{\Psi(1) \cdot(\Psi(1)+1)}{(\Psi(1)-\Psi(z))^{2}}
$$

Set $x=\Psi(1)$ and $a=\Psi(z)$. Let us recall all that we know about $|G|$ :

LEMMA 1.7. The following conditions hold:

(1) $|G|_{2}=2^{8}$;

(2) $|G|_{3}=3^{4}$;

(3) $|G|_{5}=5^{2}$; and

(4) $|G|$ is divisible by 7 .

Let $g=\frac{|G|}{2^{8} \cdot 3^{4} \cdot 5^{2}}$. Thus $g$ is an integer which is coprime to $2 \cdot 3 \cdot 5$, divisible by 7 and most importantly, $g$ can be written in the following form:

$$
g=2^{6} \cdot 3^{4} \cdot 5 \cdot 41^{2} \cdot \frac{x \cdot(x+1)}{(x-a)^{2}}
$$

COROllary 1.8. The following inequality is correct:

$$
2^{6} \cdot 3^{4} \cdot 5 \cdot 41^{2} \cdot \frac{x(x+1)}{(x+509)^{2}}<g<2^{6} \cdot 3^{4} \cdot 5 \cdot 41^{2} \cdot \frac{x(x+1)}{(x-509)^{2}}
$$


Proof. Since $|a| \leq 509$, we have the following inequality:

$$
x-509 \leq x-a \leq x+509
$$

Using this together with definition of $g$, we immediately obtain the desired result.

Let $f_{1}(x)=2^{6} \cdot 3^{4} \cdot 5 \cdot 41^{2} \cdot \frac{x(x+1)}{(x+509)^{2}}$ and $f_{2}(x)=2^{6} \cdot 3^{4} \cdot 5 \cdot 41^{2} \cdot \frac{x(x+1)}{(x-509)^{2}}$. Then Corollary 1.8 can be rewritten as:

$$
f_{1}(x)<g<f_{2}(x)
$$

Since $g$ is not divisible by either 2,3 or 5 , their powers must cancel out in (1.5). Also 2 must divide $x(x+1)$. Therefore $2^{4} \cdot 3^{2} \cdot 5$ divides $x-a$. So the natural question is: what about 41 ? Does it at all influence the picture?

Lemma 1.9. Suppose that 41 divides $x-a$. Then the following inequality holds:

$$
81|C|<g<88|C|
$$

Proof. If 41 divides $x-a$, then $2^{4} \cdot 3^{2} \cdot 5 \cdot 41$ divides $x-a$. In particular $2^{4} \cdot 3^{2} \cdot 5 \cdot 41 \leq x-a$. But $x-a \leq x+509$ and so $x \geq 29011$.

Consider the functions $f_{1}(x)$ and $f_{2}(x)$ for $x \geq 29011$. Since $f_{1}(x)$ increases on this interval, we have $f_{1}(x) \geq f_{1}(29011)>42083356$. Since $f_{2}(x)$ decreases on this interval, $f_{2}(x) \leq f_{2}(29011)<45143207$. These estimates together with (1.6) show that $42083356<g<45143207$, i.e., $81|C|<g<88|C|$.

Lemma 1.10. Suppose that 41 does not divide $x-a$. Then $41^{2}$ divides $|G|$ and $g<981|C|$.

Proof. Clearly, if $(41, x-a)=1$, then $41^{2}$ must divide $|G|$. So let us prove the inequality. Recall that $|a| \leq 509$. Suppose that $a \geq 0$. Then $2^{4} \cdot 3^{2} \cdot 5 \leq x-a \leq x$, i.e., $x \geq 720$. Consider the function $f_{2}(x)$ when $x \geq 720$. Since $f_{2}(x)$ decreases on this interval, $f_{2}(x) \leq f_{2}(720)$. This estimate together with (1.6) implies that $g<508048954$, i.e., $g<981|C|$.

If $a<0$, then from the formula (1.5) it follows that $g \leq 2^{6} \cdot 3^{4} \cdot 5 \cdot 41^{2}$. $\frac{x \cdot(x+1)}{(x+1)^{2}}$ and so $g<2^{6} \cdot 3^{4} \cdot 5 \cdot 41^{2}$, i.e., $g<85|C|$ and the result follows. 
LEMMA 1.11. $g \equiv 45523(\bmod |C|)$.

Proof. For every prime divisor $p$ of $|G|$, let $g_{p}=|G|_{p}$. Then the Theorem of Frobenius asserts that

$$
\left|\left\{h \in G \mid h^{g_{p}}=1\right\}\right| \equiv 0 \quad\left(\bmod g_{p}\right) .
$$

The left side of the congruence is nothing else but $1+\sum_{i} \frac{|G|}{\left|C_{G}\left(h_{i}\right)\right|}$, where the sum ranges over the representatives $h_{i}$ 's of conjugacy classes of nonidentity $p$-elements. Let $p \in\{2,3,5\}$. Since $|G|=g \cdot|C|$, Formula (1.7) can be rewritten in the following way:

$$
1+g \cdot \sum_{i} \frac{|C|}{\left|C_{G}\left(h_{i}\right)\right|} \equiv 0 \quad\left(\bmod g_{p}\right)
$$

In order to continue the calculations, we will need the following table:

The Orders of the Centralizers of $p$-elements

\begin{tabular}{|c|c|c|}
\hline$p$ & Class & Order of the Centralizer \\
\hline$p=2$ & $2_{1}$ & $2^{8} \cdot 3^{4} \cdot 5^{2}$ \\
& $4_{1}, 4_{2}$ & $2^{7} \cdot 3^{2} \cdot 5$ \\
& $8_{1}, 8_{2}, 8_{3}, 8_{4}$ & $2^{7} \cdot 3^{2} \cdot 5$ \\
& $8_{5}, 8_{6}$ & $2^{6}$ \\
& $16_{1}, 16_{2}, 16_{3}, 16_{4}$ & $2^{4} \cdot 5$ \\
\hline$p=3$ & $3_{1}, 3_{2}$ & $2^{4} \cdot 3^{4} \cdot 5$ \\
& $3_{3}, 3_{4}$ & $2 \cdot 3^{4}$ \\
\hline$p=5$ & $5_{1}, 5_{2}, 5_{3}, 5_{4}$ & $2^{5} \cdot 3^{2} \cdot 5^{2}$ \\
\hline
\end{tabular}

Substituting the data from the table into the Formula (1.8) for $p \in$ $\{2,3,5\}$, we obtain the following congruences:

$$
g \equiv 211 \quad\left(\bmod 2^{8}\right), g \equiv 1 \quad\left(\bmod 3^{4}\right), g \equiv 23 \quad\left(\bmod 5^{2}\right)
$$

Finally applying the Chinese Remainder Theorem, we obtain that

$$
g \equiv 45523 \quad\left(\bmod 2^{8} \cdot 3^{4} \cdot 5^{2}\right)
$$

which is precisely what we wanted to show.

LEMMA 1.12. If 41 divides $x-a$, then $g=7 \cdot 1039 \cdot 5851$. 
Proof. Since 41 divides $x-a$, Lemma 1.9 gives that $81|C|<g<$ $88|C|$. On the other hand $g \equiv 45523(\bmod |C|)$. Recall that 7 divides $g$. Putting together all this information, we obtain the unique solution: $g=7 \cdot 1039 \cdot 5851$.

COROllary 1.13. 41 does not divide $x-a$.

Proof. Assume the contrary. Then as we just proved, $g=7 \cdot 1039 \cdot 5851$ and so $|G|=2^{8} \cdot 3^{4} \cdot 5^{2} \cdot 7 \cdot 1039 \cdot 5851$. Let $Q \in \operatorname{Syl}_{1039}(G)$ and $N=N_{G}(Q)$. By Sylow Theorem, $|G: Q| \equiv|N: Q|(\bmod 1039)$. Thus $|N: Q| \equiv 418$ $(\bmod 1039)$. Since the centralizer of $Q$ is a $\{2,3,5\}^{\prime}$-group and $1038=$ $2 \cdot 3 \cdot 173$, we obtain that $|N: Q|$ divides $2 \cdot 3 \cdot 7 \cdot 5851$. Therefore there exist integers $t \geq 1, r \geq 1$ such that

$$
(1039 t+418) r=2 \cdot 3 \cdot 7 \cdot 5851
$$

Solving it modulo 1039, we obtain that $r \equiv 51(\bmod 1039)$. If $r>51$, then the left side of (1.9) becomes strictly larger than the right side. Therefore $r=51$, which is a contradiction.

Therefore we are now in the conditions of Lemma 1.10. So let us summarize all that we know about $g$ :

$$
g<981|C|, g \equiv 45523 \quad(\bmod |C|) \text { and } g \equiv 0 \quad\left(\bmod 7 \cdot 41^{2}\right)
$$

Putting the last two together with the help of the Chinese Remainder Theorem, we obtain that $g \equiv 4651130323\left(\bmod 7 \cdot 41^{2} \cdot|C|\right)$. But this means that $g>8972|C|$, which is an obvious contradiction proving the result.

\section{REFERENCES}

[Co] M.J. Collins, Representations and characters of finite groups, Cambridge University Press (1990).

[GLS] D. Gorenstein, R. Lyons and R. Solomon, The Classification of the Finite Simple Groups, Number 1, Amer. Math. Soc. Surveys and Monographs, 40, \# 1 (1995).

[GLS2] The Classification of the Finite Simple Groups, Number 1, Amer. Math. Soc. Surveys and Monographs, 40, \# 6 (to be published).

[F1] P. Fong, A Characterization of the finite simple groups $P \operatorname{Sp}(4, q), G_{2}(q),{ }^{2} D_{4}(q)$. Part 2, Nagoya Math. J., 39 (1970), 37-79.

[F2] P. Fong, Unpublished Notes.

[FW] P. Fong and W.J. Wong, A Characterization of the finite simple groups PSp $(4, q)$, $G_{2}(q),{ }^{2} D_{4}(q)$. Part 1, Nagoya Math. J., 36 (1969), 143-184. 
[Is] I. Martin Isaacs, Character Theory of Finite Simple Groups, Academic Press, New York, 1976.

[L] R. Lyons, Evidence for a new simple group, J. Algebra, 20 (1972), 540-569.

Department of Mathematics

Hill Center-Busch Campus

Rutgers, The State University of New Jersey

110 Frelinghuysen $R d$

Piscataway, NJ 08854-8019

U.S.A.

innako@math.rutgers .edu 